\title{
Development of Ontology and 3D Software for the Diseases of Spine
}

\author{
Seungbock Lee, ${ }^{1}$ Sangho Lee, ${ }^{1}$ Dongmin Seo, ${ }^{1}$ Kwan-Hee Yoo, ${ }^{2}$ and Sukil Kim ${ }^{3}$ \\ ${ }^{1}$ Software Research Center, Korea Institute of Science and Technology Information, Daejeon 305-806, Republic of Korea \\ ${ }^{2}$ Department of Digital Information Convergence, Chungbuk National University of Korea, Cheongju 361-763, Republic of Korea \\ ${ }^{3}$ College of Medicine, The Catholic University of Korea, Seoul 137-701, Republic of Korea
}

Correspondence should be addressed to Sukil Kim; sikimmd@gmail.com

Received 28 August 2014; Accepted 18 November 2014

Academic Editor: Seungmin Rho

Copyright (C) 2015 Seungbock Lee et al. This is an open access article distributed under the Creative Commons Attribution License, which permits unrestricted use, distribution, and reproduction in any medium, provided the original work is properly cited.

\begin{abstract}
KISTI is carrying out an e-Spine project for spinal diseases to prepare for the aged society, so-called NAP. The purpose of the study is to build a spine ontology that represents the anatomical structure and disease information which is compatible with simulation model of KISTI. The final use of the ontology includes diagnosis of diseases and setting treatment directions by the clinicians. The ontology was represented using 3D software. Twenty diseases were selected to be represented after discussions with a spine specialist. Several ontology studies were reviewed, reference books were selected for each disease and were organized in MS Excel. All the contents were then reviewed by the specialists. Altova SemanticWorks and Protégé were used to code spine ontology with OWL Full model. Links to the images from KISTI and sample images of diseases were included in the ontology. The OWL ontology was also reviewed by the specialists again with Protégé. We represented unidirectional ontology from anatomical structure to disease, images, and treatment. The ontology was human understandable. It would be useful for the education of medical students or residents studying diseases of spine. But in order for the computer to understand the ontology, a new model with OWL DL or Lite is needed.
\end{abstract}

\section{Introduction}

KISTI (Korean Institute of Science and Technology Information) has been studying a National Agenda Project (NAP) for developing elderly human body model for treatment and rehabilitation of age-related spinal disorders. The purpose of the study is to build a virtual human spine as a simulation model through mathematical modeling to use in virtual experiment instead of real human spine. Accurate diagnosis and treatment of spinal diseases were expected from the project. We tried to develop the spinal ontology which contains information on spine and the related diseases for the success of the main project.

Ontology is systemized process accomplished by using computers for building a model which present the recognizable concepts and the relations between them [1]. Neches et al. (1991) defined ontology as "basic terms and relations comprising the vocabulary of a topic area as well as the rules for combining terms and relations to define extensions to the vocabulary" [2] and Gruber (1993) defined it as "an explicit specification of a conceptualization" [3].

This study focused on developing spinal ontology with frequently occurring spinal diseases in Koreans. It contains anatomy of spine, method of treatment, cause, and classification information related with spine. Further, the spinal ontology can be linked to the simulation model for education of medical students and for the physicians and biomedical engineers by offering the necessary information in their fields.

\section{Method}

The authors are composed of a wide range of professional researchers, medical informatics, computer professionals, and clinical experts such as nurses, a neurosurgeon, and 2 imaging specialists. The research was conducted in 5 phases (Figure 1): (1) review related to existing ontology for the construction of the model, (2) selecting the spine related diseases and the subject of the research at the same time, 


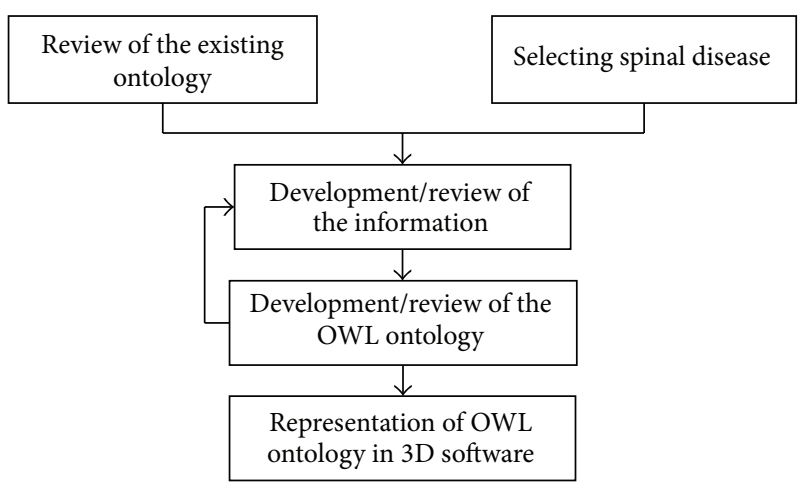

FIgURE 1: Process of building spine ontology.

(3) developing/reviewing spinal ontology, (4) creating OWL ontology in accordance with the clinician's feedback, and (5) Reviewing the OWL ontology by the specialists. In addition, the ontology was represented by $3 \mathrm{D}$ image software for the easy understanding.

2.1. Review of the Existing Ontology. We reviewed results of ontology project using Protégé which were led in 1987 as a public project at Stanford University [4]. Among their results, we gathered information that is necessary for spine ontology after analyzing the ontology of rat anatomy and classification of diseases.

2.2. Selecting Spinal Diseases. The spinal diseases were selected based on the following three criteria. First, the disease has to be one of the highly occurring spinal diseases among Koreans. Second, the disease must occur in a specific area of the spine rather than throughout the whole spine. This makes it possible for the ontology to provide information that is suitable to the characteristics of the diseases among Koreans. Also, it is much easier to link with the simulation model of KISTI which is made according to Korean human being. Last, the OWL ontology of the disease should be able to be expressed on the computer so that it can be used in clinics or medical schools for education.

2.3. Development/Review of the Information. The developed ontology was organized according to the diseases with Microsoft Excel. The anatomic definitions that consist of ontology were referenced from a medical dictionary [5] and the disease related information was extracted from publications recommended by clinicians [6]. We classified anatomical information into two categories; anatomical location and anatomic properties were represented in OWL ontology (Table 1).

Disease related information was classified into five categories as shown in Table 2; anatomical location, property of the disease, symptom/sign, method of treatment, and image were represented in OWL ontology.

The spinal ontology was reviewed by a neurosurgeon and two imaging specialists. Sample images of diseases, CT or
TABLE 1: Anatomical information represented in the ontology.

\begin{tabular}{llc}
\hline Entry & Ontology & OWL expression \\
\hline Location & Anatomical location & spine: isPartOf \\
\hline \multirow{4}{*}{ Properties } & Part name & rdfs: label \\
& $\begin{array}{l}\text { Anatomical classification } \\
\text { Standard code for the }\end{array}$ & rdfs: subClassOf \\
& $\begin{array}{l}\text { structure } \\
\text { Definition and } \\
\text { description of the } \\
\text { structure }\end{array}$ & spine: KOSTOM \\
& & spine: definition \\
& spine: description
\end{tabular}

M.R.I, were collected during the study period in Seoul St. Mary's Hospital and linked to the diseases in the ontology.

2.4. Development/Review of the OWL Ontology. Spinal OWL ontology was built based on OWL Full model which is a standard ontology language developed by W3C (World Wide Web Consortium). Both Altova Semantic Works and Protégé were used to build and review the OWL ontology.

In the process of OWL representation, we tried to determine the level of expression in classes (resource object) or individuals (literal object). For example, if the disease related information is "herniated nucleus pulposus", 〈herniated nucleus pulposus〉 is identified as superclass and 〈cervical intervertebral disc herniation $\rangle,\langle$ lumbar intervertebral disc herniation〉, and 〈thoracic intervertebral disc herniation〉 which are classified under herniated nucleus pulposus are identified as subclass according to their location. Each class contains additional information related to their occurring region.

2.5. Representation of OWL Ontology in 3D Software. The contents of OWL ontology on spine were represented by using 3D image S/W. The software has three modules: 3D rendering module, OWL query module, and the module for showing disease information that comes from the ontology. The users may select a part of spine image of question. Then a list of the diseases from the OWL spine ontology file will appear through OWL query operation. When a disease among the list is selected, the query module searches the disease related information such as causes, symptoms, diagnoses, treatment, complication, and image of the disease.

\section{Result}

3.1. Selected Spinal Diseases. The list of 20 selected diseases is presented in Table 3. If scientific papers were referred in addition to text book, they were added as references in the table.

3.2. Development of the Ontology. Figure 2 presents Protégé OWL ontology graph created from the spinal ontology of the twenty selected diseases. The anatomical class represents the entire structure that composes the spine. There are 50 classes; 1 vertebral column, 5 vertebrae, 33 vertebrae, and other 11 materials of spine. Each class has 6 properties. To 
TABLE 2: Diseases related information represented in ontology.

\begin{tabular}{|c|c|c|}
\hline Entry & Ontology & OWL expression \\
\hline Location & Anatomical disease location & spine: hasSite \\
\hline \multirow{9}{*}{ Disease properties } & Name of disease & rdfs: label \\
\hline & Classification of diseases & rdfs: subClassOf \\
\hline & Apply the standard code & spine: KOSTOM \\
\hline & Definition of disease & spine: definition \\
\hline & 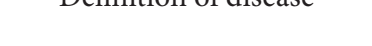 & spine: description \\
\hline & Clinical diagnosis & spine: diagnosis \\
\hline & Cause & spine: hasCause \\
\hline & Concomitant diseases & spine: hasConcomitantDisease \\
\hline & Complication & spine: hasComplication \\
\hline \multirow{3}{*}{ Symptom/sign } & Symptom & spine: hasSymptom \\
\hline & & spine: causeOfSymptom \\
\hline & Sign & spine: hasSign \\
\hline \multirow{3}{*}{ Treatment } & Surgical treatment & spine: hasSurgicalTreatment \\
\hline & Nonsurgical treatment & spine: hasNonSurgicalTreatment \\
\hline & Conservative treatment & spine: hasTreatmentConservative \\
\hline \multirow{2}{*}{ Image } & Preoperative image & spine: hasImageBeforeTx \\
\hline & Postoperative image & spine: hasImageAfterTx \\
\hline
\end{tabular}

TABLE 3: The twenty selected diseases.

Atlas fracture

Degenerative marrow change (Modic type change)

Grading of lumbar disc degeneration [7]

Hangman's fracture

HNP (herniation of nucleus pulposus) [8]

Infectious spondylitis

Kyphosis

Meningocele [9]

Odontoid process fracture

OPLL (ossification of posterior longitudinal ligament)

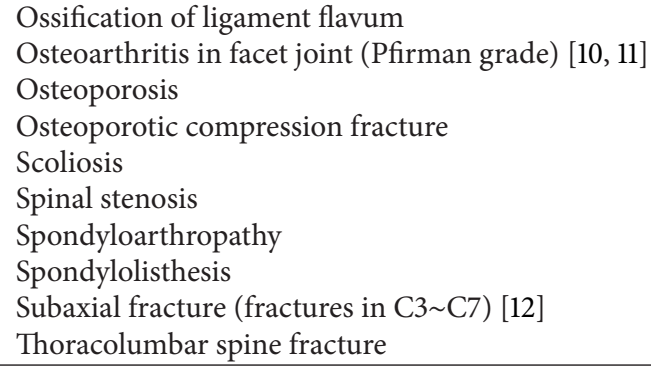

express a sentence " $\mathrm{C} 1$ cervical is part of cervical vertebra" in OWL Full model, we defined anatomical structures "Cl" and "cervical vertebra" as a class and 〈is a part of as a property representing a predicate. 〈Disease〉 was defined as a superclass and the 20 selected diseases were treated as classes. As a result, 21 classes were formed for the concept of diseases and each class has 18 properties.

More than 100 images that were collected were linked to 20 diseases, one per each. The predicates that link images to the other part of ontology include 〈hasImageBeforeTx $\rangle$ and $\langle$ hasImageAfterTx $\rangle$.

3.3. Representation of Spine Ontology in 3D Software. The user interface is composed of three parts (Figure 3). (1) 3D rendering part: the users can rotate, move, zoom in, and zoom out the whole spine on the left upper part of the window and the detail of the selected anatomical structure in a large scale is displayed on the bottom left window with its textual relationships. (2) The list of diseases: the diseases that are related with the anatomic site on the $3 \mathrm{D}$ rendering part are displayed on the right upper part of the window following the ontological relationship between anatomic sites to diseases.
(3) The details of the disease specific information: when a disease is selected in the upper right window, the information of the disease is listed on the bottom right window, which includes disease properties, treatments, and the related diagnostic images. If there are textual descriptions on the properties, they are also displayed on the bottom right window.

\section{Conclusions}

We built the ontology of spine with links to the cause, symptoms, method of treatment of highly occurring spinal disease among Koreans, and anatomical information.

The completed spinal ontology expresses anatomical connection of the parts of spine and their vertical relationships as well as information on the diseases in the spine. It is easy to understand the structure and the diseases of spine by conceptualizing the anatomical structure of spine and show them in 3D images.

This study was completed by the use of literal object of OWL Full model by expressing the contents of the main reference dictionary and publications about spine literally. But in order for the computer to interpret the ontology, a new 


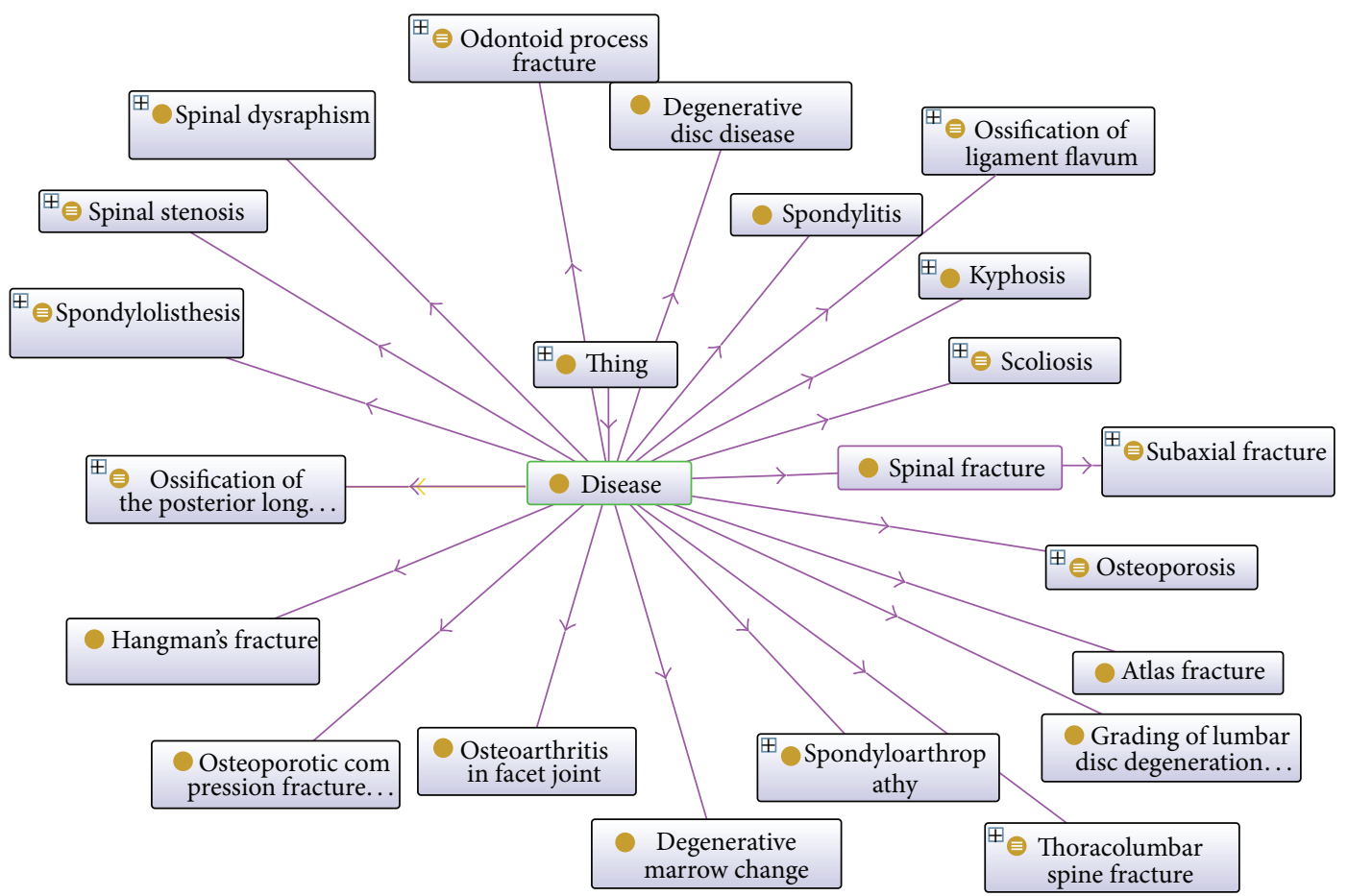

Figure 2: List of the spinal diseases shown in Protégé.

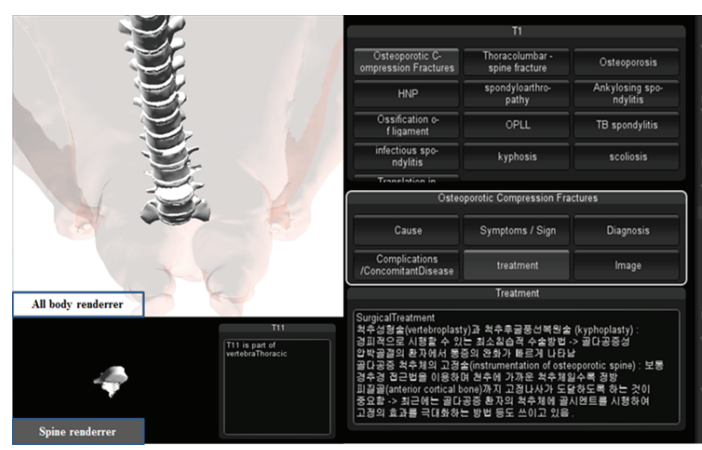

FIGURE 3: User interface of the proposed system.

model with OWL DL or Lite is needed. Further studies need to include the process of the transformation of literal object into resource object through the structuralization process of items completed by literal object, further systematizing the concept. In addition, the review of class and property is necessary to show the anatomical information of spine and information of diseases specifically. Also, the studies about methods which offer visual information are related to simulation model of KISTI.

\section{Conflict of Interests}

The authors declare that there is no conflict of interests regarding the publication of this paper.

\section{References}

[1] R. Mizoguchi, The Next Generation Web and Critical Technology of Knowledge Processing, Dooyangsa, 2009.

[2] R. Neches, R. Fikes, T. Finin et al., "Enabling technology for knowledge sharing," AI Magazine, vol. 12, no. 3, pp. 36-56, 1991.

[3] T. R. Gruber, "A translation approach to portable ontology specifications," Knowledge Acquisition, vol. 5, no. 2, pp. 199-220, 1993.

[4] J. H. Gennari, M. A. Musen, R. W. Fergerson et al., "The evolution of Protégé: an environment for knowledge-based systems development," International Journal of Human Computer Studies, vol. 58, no. 1, pp. 89-123, 2003.

[5] J. Ji, Stedman's Medical Dictionary, Koonja Publishing, 2006.

[6] Society TKSN, The Textbook of Spine, 2008.

[7] C. W. A. Pfirrmann, A. Metzdorf, M. Zanetti, J. Hodler, and N. Boos, "Magnetic resonance classification of lumbar intervertebral disc degeneration," Spine, vol. 26, no. 17, pp. 18731878, 2001.

[8] M. T. Modic and J. S. Ross, "Lumbar degenerative disk disease," Radiology, vol. 245, no. 1, pp. 43-61, 2007.

[9] S. Jeffrey, K. R. M. Ross, B. Bryson et al., Diagnostic Imaging: Spine, Amirsys, 2010.

[10] M. Pathria, D. J. Sartoris, and D. Resnick, "Osteoarthritis of the facet joints: accuracy of oblique radiographic assessment," Radiology, vol. 164, no. 1, pp. 227-230, 1987.

[11] D. Weishaupt, M. Zanetti, N. Boos, and J. Hodler, "MR imaging and CT in osteoarthritis of the lumbar facet joints," Skeletal Radiology, vol. 28, no. 4, pp. 215-219, 1999.

[12] J. W. M. van Goethem, L. van den Hauwe, P. Parizel, and A. L. Baert, Spinal Imaging: Diagnostic Imaging of the Spine and Spinal Cord, Springer, 2007. 

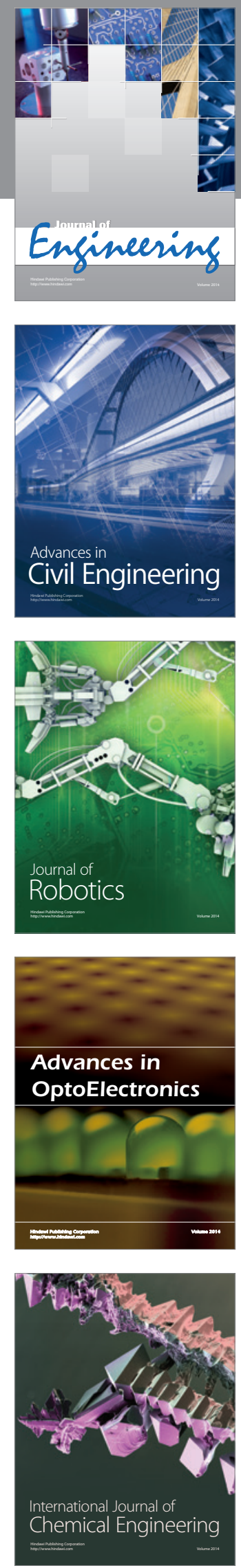

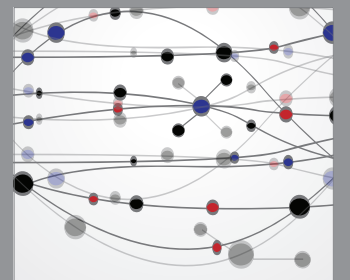

The Scientific World Journal
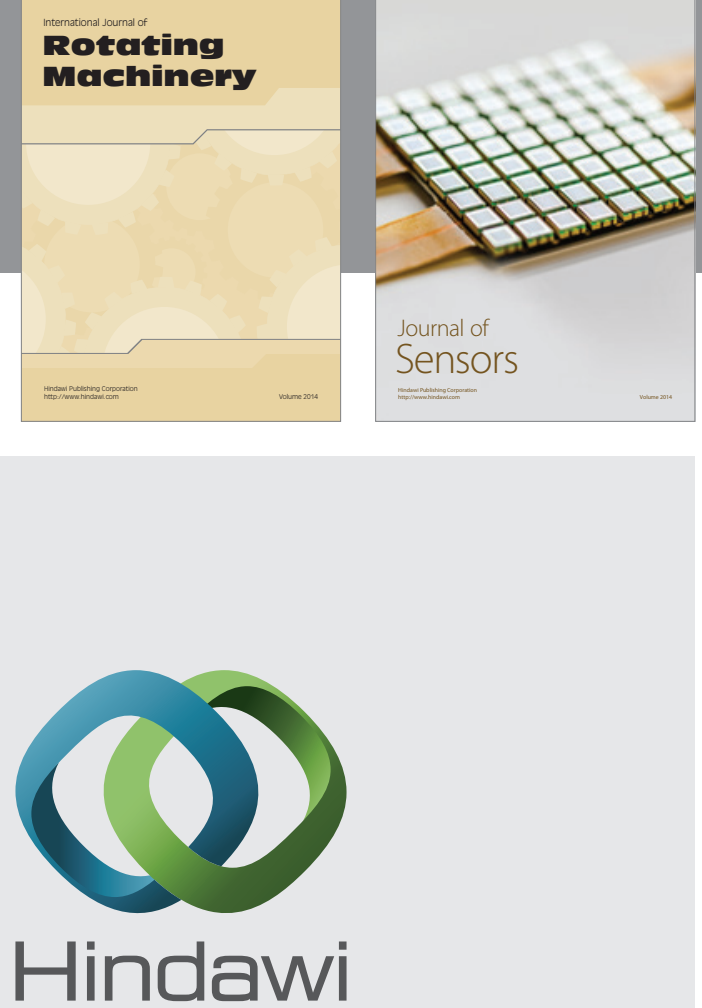

Submit your manuscripts at http://www.hindawi.com
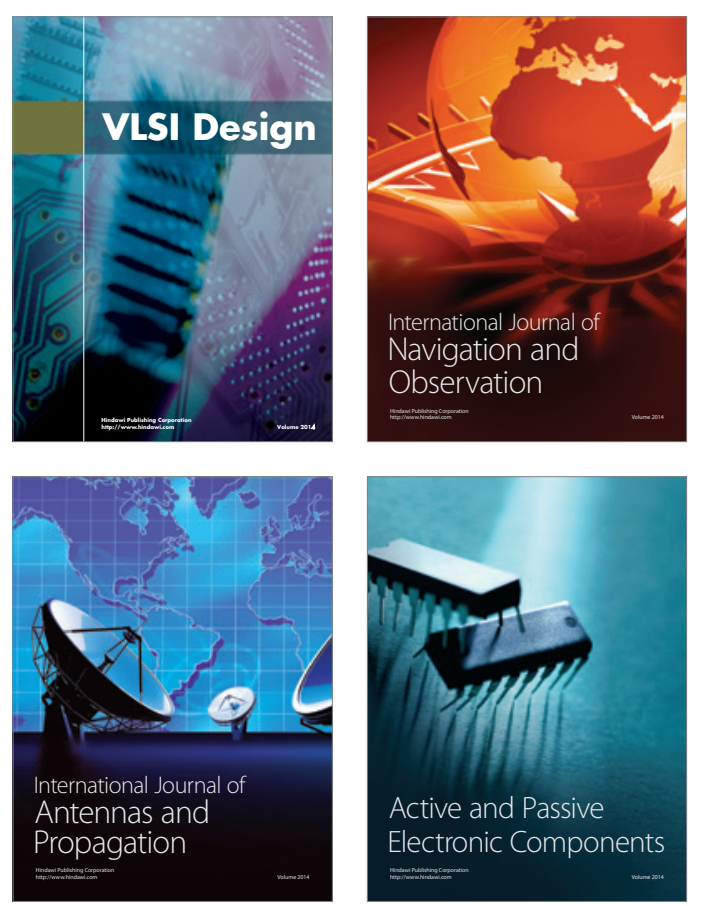
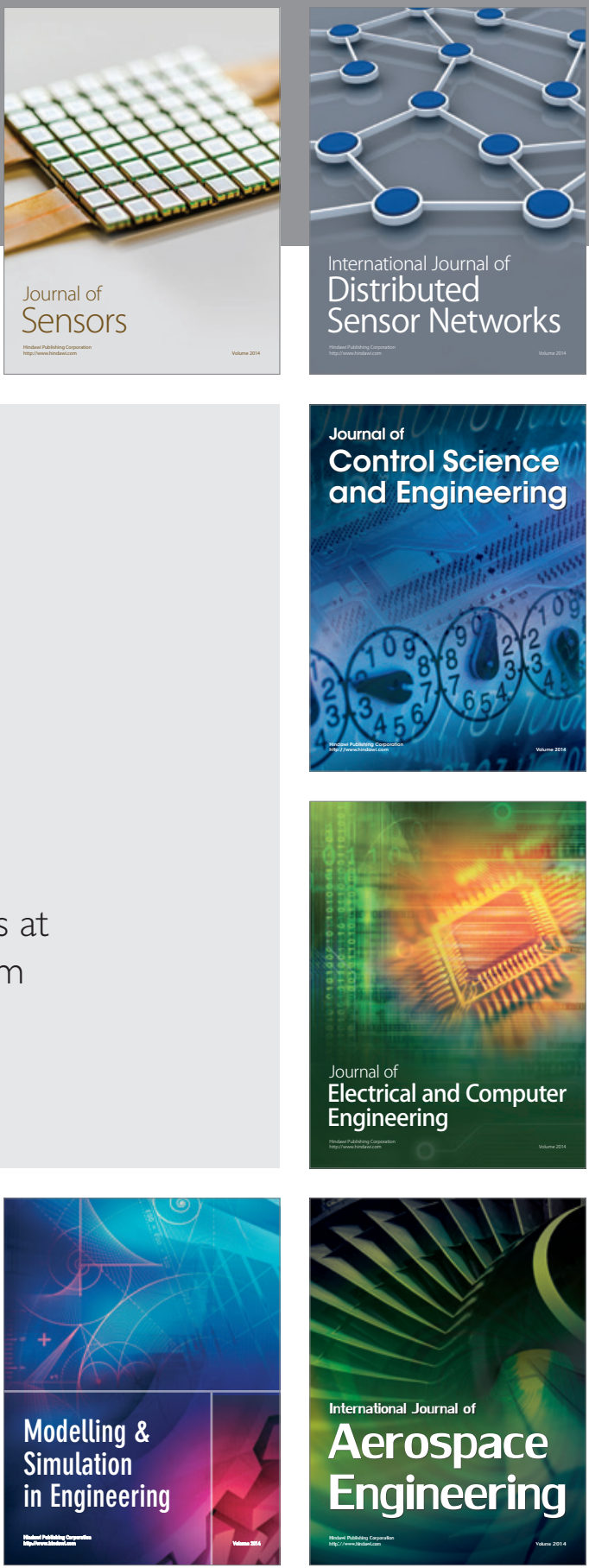

Journal of

Control Science

and Engineering
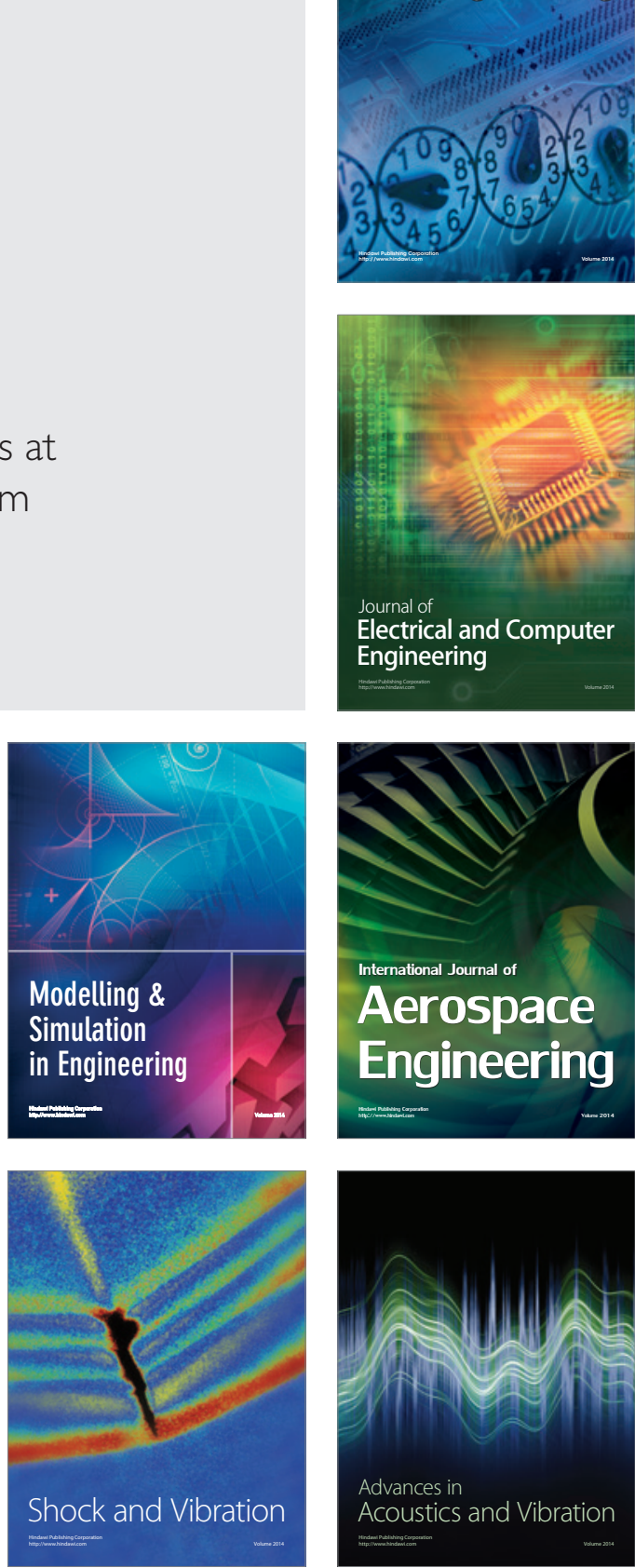